\title{
Optimum Control for Nonlinear Dynamic Radial Deformation of Turbine Casing with Time-Varying LSSVM
}

\author{
Cheng-Wei Fei, ${ }^{1,2}$ Guang-Chen Bai, ${ }^{3}$ Wen-Zhong Tang, ${ }^{1}$ and Yatsze Choy ${ }^{2}$ \\ ${ }^{1}$ School of Computer Science and Engineering, Beijing University of Aeronautics and Astronautics, Beijing 100191, China \\ ${ }^{2}$ Department of Mechanical Engineering, The Hong Kong Polytechnic University, Hung Hom, Kowloon, Hong Kong \\ ${ }^{3}$ School of Energy and Power Engineering, Beijing University of Aeronautics and Astronautics, Beijing 100191, China
}

Correspondence should be addressed to Cheng-Wei Fei; feicw544@163.com

Received 18 October 2014; Accepted 9 February 2015

Academic Editor: Peter Majewski

Copyright (C) 2015 Cheng-Wei Fei et al. This is an open access article distributed under the Creative Commons Attribution License, which permits unrestricted use, distribution, and reproduction in any medium, provided the original work is properly cited.

\begin{abstract}
With the development of the high performance and high reliability of aeroengine, the blade-tip radial running clearance (BTRRC) of high pressure turbine seriously influences the reliability and performance of aeroengine, wherein the radial deformation control of turbine casing has to be concerned in BTRRC design. To improve BTRRC design, the optimum control-based probabilistic optimization of turbine casing radial deformation was implemented using time-varying least square support vector machine (TLSSVM) by considering nonlinear material properties and dynamic thermal load. First the T-LSSVM method was proposed and its mathematical model was established. And then the nonlinear dynamic optimal control model of casing radial deformation was constructed with T-LSSVM. Thirdly, through the numerical experiments, the T-LSSVM method is demonstrated to be a promising approach in reducing additional design samples and improving computational efficiency with acceptable computational precision. Through the optimum control-based probabilistic optimization for nonlinear dynamic radial turbine casing deformation, the optimum radial deformation is $7.865 \times 10^{-4} \mathrm{~m}$ with acceptable reliability degree 0.9956 , which is reduced by $7.86 \times 10^{-5} \mathrm{~m}$ relative to that before optimization. These results validate the effectiveness and feasibility of the proposed T-LSSVM method, which provides a useful insight into casing radial deformation, BTRRC control, and the development of gas turbine with high performance and high reliability.
\end{abstract}

\section{Introduction}

Blade-tip radial running clearance (BTRRC) of high pressure turbine (HPT) seriously influences the performance and reliability of gas turbine [1]. Large BTRRC causes the decline of aeroengine performance just like low working efficiency and thrust, high specific fuel consumption, and so forth, while small BTRRC results in the friction fault between bladetip and casing [2,3]. Hence, BTRRC has to hold a reasonable value under operating conditions. Hereinto, casing radial deformation is one important factor of directly effecting on BTRRC [4]. The optimum control of casing radial deformation should be done to make BTRRC more reasonable. In advanced propulsive systems, turbine casing bears vast thermal load and pressure difference under working conditions due to high temperature gradient and high rotational velocity $[5,6]$. A significant objective of the probabilistic optimal control of casing radial deformation is to seek an optimum radial deformation to offset the radial deformations of turbine disk and blade under satisfying an acceptable reliability degree. Therefore, an effective optimization method-based probabilistic analysis is required for the optimum control of turbine casing radial deformation.

With the growth in the computing power of current computers, finite element (FE) method has become a common and important technique in product development process, which has been applied to the design analysis of aeroengine components like stress analysis, thermal analysis, vibration analysis, and fatigue life estimate [7-10]. Excessive computational cost and impractical runtime are confronted when FE method is adopted in the probabilistic optimal design of complex structure like turbine casing because of too large computational loads for nonlinear analysis, dynamic analysis, and multiple loop computations. To reduce the runtime, one 
alternative is to construct a simple surrogate model (also called as response surface method, RSM) to approximate the response of FE solver [5]. The surrogate model expresses the relationship between the objective or constraint functions and design variables by simple equations, which only cost a small number of FE analyses in order to significantly reduce computing time. The typical surrogate models include polynomial response surface method [11-16] and support vector machine (SVM) [17-21], and so forth. Polynomial response surface model uses least squares regression analysis to fit low-order polynomials to a set of experimental data $[15,16]$. Currently, SVM as an intelligent statistical learning method and implicit performance function has been employed to the reliability analysis and optimal design of complex structure instead of the polynomial response surface method, because SVM holds the advantages of small training sample, high computational accuracy, and efficiency. To further improve the computing speed for building SVM model, the least square SVM (LSSVM) was developed and proved to be an effective method for structure reliability design-based steady analysis [18]. However, it is difficult that the LSSVM is directly applied to the nonlinear dynamic optimal analysis of complex structure with time-varying characteristics [22, 23].

To accomplish the optimum control for nonlinear dynamic radial deformation of turbine casing with LSSVM, the time-varying LSSVM (T-LSSVM) method with high efficiency and high precision is presented. And the mathematical model of T-LSSVM is established and verified to be effective and feasible by numerical experimentation. Finally, the optimum control-based probabilistic optimization for nonlinear dynamic radial deformation of turbine casing was performed with the proposed T-LSSVM, subject to constraints on reliability degree and other practical conditions with nonlinear material property and dynamic thermal load.

\section{Time-Varying LSSVM (T-LSSVM)}

During aeroengine working, thermal stress and pressure difference are of variation. Hence, the probabilistic optimization of casing radial deformation needs a time-varying (or dynamic) optimization method. In this section, the TLSSVM method is proposed based on LSSVM for dynamic probabilistic optimization.

2.1. Least Square Support Vector Machine. SVM is an important surrogate model based on intelligent statistical learning theory [24] and holds high computational precision and efficiency. LSSVM adopts linear equation in high-dimensional space replacing quadratic programming problem in lowdimensional space to improve computational efficiency by taking the term of squared error as the objective function and regarding an equation as the constraints $[17,18]$. The mathematical model of LSSVM is summarized as follows.

From a certain kind of assumed distribution $P(\mathbf{x}, y)(\mathbf{x} \in$ $\mathbf{R}^{n}$ and $\left.y \in \mathbf{R}\right)$, the sampling points $\left\{\left(\mathbf{x}_{i}, \mathbf{y}_{i}\right)\right\}_{i=1,2, \ldots, l}$ are generated, and a set of functions $\mathbf{F}$ which maps a point in the space $\mathbf{R}^{n}$ onto the space $\mathbf{R}$ is denoted by

$$
\mathbf{F}=\left\{f(\mathbf{x}, \boldsymbol{\omega}), \boldsymbol{\omega} \in \Lambda \mid f: \mathbf{R}^{n} \longrightarrow \mathbf{R}\right\},
$$

in which $\Lambda$ is a set of parameters and $\omega$ is an undetermined parameter vector.

The regression object is to find a function $f \in F$ which makes (1) have the lowest expected risk:

$$
\begin{aligned}
& R(f)=\int l(y-f(\mathbf{x}, \boldsymbol{\omega})) \mathrm{d} P(\mathbf{x}, y), \\
& \text { s.t. }(y-f(\mathbf{x}, \boldsymbol{\omega}))=\max \{0,|y-f(\mathbf{x}, \boldsymbol{\omega})|-\varepsilon\},
\end{aligned}
$$

in which $l(y-f(\mathbf{x}, \boldsymbol{\omega}))$ is an error function [21] $\varepsilon>0$. Function $f$ can be determined by the following method.

For the nonlinear regression, each sampling point is mapped by a nonlinear function $\varphi$ onto the high-dimensional space to conduct the linear regression, and then the original space nonlinear regression effect is attained. Thus, the function $f$ is rewritten as

$$
f(\mathbf{x}, \boldsymbol{\omega})=\boldsymbol{\omega} \cdot \boldsymbol{\varphi}(\mathbf{x})+b .
$$

Obviously, the problem of solving the regression function can be transformed into obtaining the following optimal solution:

$$
\begin{array}{ll}
\min & \frac{1}{2}\|\boldsymbol{\omega}\|^{2}, \\
\text { s.t. } & \left|\boldsymbol{\omega} \cdot \boldsymbol{\varphi}\left(\mathbf{x}_{i}\right)+b-y_{i}\right|<\varepsilon \quad i=1,2, \ldots, l .
\end{array}
$$

Considering the permissible errors, two slack variables $\xi_{i}$ and $\xi_{i}^{*}$ are introduced, and the optimization function is

$$
\begin{array}{ll}
\min & \frac{1}{2}\|\boldsymbol{\omega}\|^{2}+\gamma \sum_{i=1}^{l}\left(\xi_{i}+\xi_{i}^{*}\right) \\
\text { s.t. } & \boldsymbol{\omega} \cdot \boldsymbol{\varphi}\left(\mathbf{x}_{i}\right)+b-y_{i} \leq \xi_{i}^{*}+\varepsilon \\
& y_{i}-\boldsymbol{\omega} \cdot \boldsymbol{\varphi}\left(\mathbf{x}_{i}\right)-b \leq \xi_{i}^{*}+\varepsilon \\
& \xi_{i}, \xi_{i}^{*} \geq 0, \quad i=1,2, \ldots, l,
\end{array}
$$

where $\gamma$ is a penalty coefficient.

For obtaining the solution of this quadratic program, the Lagrange function is introduced:

$$
\begin{aligned}
L\left(\boldsymbol{\omega}, b, \mathbf{a}, \mathbf{a}^{*}\right)= & \min \frac{1}{2}\|\boldsymbol{\omega}\|^{2}+\gamma \sum_{i=1}^{l}\left(\xi_{i}+\xi_{i}^{*}\right) \\
& -\sum_{i=1}^{l} a_{i}\left(\xi_{i}+\varepsilon-y_{i}+\boldsymbol{\omega} \cdot \boldsymbol{\varphi}\left(\mathbf{x}_{i}\right)+b\right) \\
& +\sum_{i=1}^{l} a_{i}^{*}\left(\xi_{i}^{*}+\varepsilon+y_{i}-\boldsymbol{\omega} \cdot \boldsymbol{\varphi}\left(\mathbf{x}_{i}\right)-b\right) \\
& -\sum_{i=1}^{l} \eta_{i}\left(\xi_{i}+\xi_{i}^{*}\right),
\end{aligned}
$$

where $a_{i}, a_{i}^{*} \geq 0, i=1,2, \ldots, l$.

In the optimization process, a kernel function $\psi\left(\mathbf{x}_{i}, \mathbf{x}_{j}\right)$ is used to replace the inner product $\left\langle\varphi\left(\mathbf{x}_{i}\right), \varphi\left(\mathbf{x}_{j}\right)\right\rangle$ in higher 
dimensional space, where the Lagrange duality problem is expressed by

$$
\begin{array}{ll}
\min _{\mathbf{a}, \mathbf{a}^{*}} & {\left[\frac{1}{2} \sum_{i, j=1}^{l}\left(a_{i}^{*}-a_{i}\right)\left(a_{j}^{*}-a_{j}\right) \psi\left(\mathbf{x}_{i}, \mathbf{x}_{j}\right)\right.} \\
& \left.+\varepsilon \sum_{i=1}^{l}\left(a_{i}^{*}-a_{i}\right)-\sum_{i=1}^{l} y_{i}\left(a_{i}^{*}-a_{i}\right)\right], \\
\text { s.t. } \quad & \sum_{i=1}^{l}\left(a_{i}^{*}-a_{i}\right)=0 \quad\left(0 \leq a_{i}, a_{i}^{*} \leq \gamma ; i=1,2, \ldots, l\right) .
\end{array}
$$

After getting the optimized solution $\bar{a}_{i}, \bar{a}_{i}^{*}$, and $\bar{b}$, the regression estimating function (LSSVM model) is

$$
\begin{gathered}
f(\mathbf{x})=\sum_{\mathbf{x}_{i} \in \mathrm{SV}}\left(\bar{a}_{i}-\bar{a}_{i}^{*}\right) \psi\left(\mathbf{x}, \mathbf{x}_{i}\right)+\bar{b}, \\
\mathbf{x}=\left[\mathbf{x}_{1}, \mathbf{x}_{2}, \ldots, \mathbf{x}_{l}\right], \quad \mathbf{x}_{i}=\left[x_{i 1}, x_{i 2}, \ldots, x_{i r}\right]^{T},
\end{gathered}
$$

where SV is a set of support vectors for a given sample set; $i=1,2, \ldots, l, l$ is the number of sample values; $r$ is the number of random variables.

2.2. Time-Varying LSSVM. For time-varying probabilistic optimization problem, the response of each calculation is a stochastic process so that it is difficult to finish the probabilistic analysis of complex structure with response surface method. In the face of this situation, conventional approaches fit a lot of response surface models in this time-varying process to select one reasonable response at one time point as the computational point of probabilistic optimization. However, in all of the loop computations of probabilistic optimization, it is difficult to make this computational point reasonable and feasible, so that the computational accuracy of probabilistic design is low. To resolve this issue using LSSVM, this paper advances the T-LSSVM method for the dynamic probabilistic optimization of turbine casing radial deformation. T-LSSVM is used to calculate a single extreme value rather than a series of dynamic output responses under different input samples within a time domain $[0, T]$, which is equivalent to transform a stochastic process of output response into a random variable. In each stochastic analysis, the random variable as the response can be ensured to be the most reasonable and effective, which can pledge the analytical precision. And then the T-LSSVM model is established based on these data for probabilistic optimization. The procedure of probabilistic optimization with T-LSSVM is drawn as follows.

(1) Establish the FE model of structure and select reasonable parameters (dynamic load, constraint conditions, time domain, etc.).

(2) Extract the samples of input and output variables and define the maximum output responses for each stochastic analysis within time domain.
(3) Fit the response surface function of T-LSSVM to these samples.

(4) Apply the T-LSSVM function of to accomplish structural dynamic probabilistic optimization.

Obviously, T-LSSVM is promising to reduce computing cost and enhance calculation efficiency. For probabilistic analysis of turbine casing radial deformation, the maximum deformation may be considered as the output response of $\mathrm{T}$ LSSVM for optimum control during the time domain $[0, T]$, because the time-varying deformation of turbine casing is secure in $[0, T]$ as long as the maximum response is safe at the time point.

According to the basic thought of T-LSSVM, the data set with the maximum output response and input random variables is obtained through a number of stochastic analyses in time domain $[0, T]$. With the $j$ th input samples $x_{j}$, the extremum of output response $Y_{i}\left(t, \mathbf{x}_{i}\right)$ is $Y_{i, \max }\left(\mathbf{x}_{i}\right)$ within time domain $[0, T]$. The data set $\left\{Y_{i, \max }\left(\mathbf{x}_{i}\right): i=1,2, \ldots, l\right\}$ consisting of the maximum output responses is used to fit the extremum response curve $Y(\mathbf{x})$ :

$$
Y(\mathbf{x})=f(\mathbf{x})=\left\{Y_{i, \max }\left(\mathbf{x}_{i}\right): i=1,2, \ldots, l\right\},
$$

where $f(\mathbf{x})$ is a response surface function, called T-LSSVM function (or model) here.

In fact, for dynamic probabilistic design the T-LSSVM function is a key factor because a valid T-LSSVM function is conductive to enhance the efficiency and precision of probabilistic design. If the T-LSSVM function replacing FE model is applied to structural dynamic probabilistic design, this method is called T-LSSVM method, which belongs to the global response surface method. Based on (8), the T-LSSVM function is built as follows:

$$
\begin{aligned}
Y(\mathbf{x}) & =\operatorname{Max}_{j} f\left(\mathbf{x}_{j}\right) \\
& =\operatorname{Max}_{j}\left\{\sum_{\mathbf{x}_{i j} \in S \mathrm{~V}}\left(\bar{a}_{i j}-\bar{a}_{i j}^{*}\right) \psi\left(\mathbf{x}_{j}, \mathbf{x}_{i j}\right)+\bar{b}_{j}\right\}, \quad j \in N,
\end{aligned}
$$

where $j=1,2, \ldots, n, n$ is the number of fitted LSSVM functions of in one stochastic analysis process within $[0, T]$.

\section{Problem Formulation}

Casing radial deformation under gas turbine operation is an important component influencing BTRRC variation [1$3,25,26$ ]. In BTRRC control, small casing radial deformation produces a small BTRRC, which degrades the reliability of gas turbine, while large casing radial deformation increases BTRRC and lessens the performance of gas turbine. Clearly, casing radial deformation should be potentially controlledbased probabilistic optimization subject to a reasonable reliability degree.

3.1. FE Model of Turbine Casing. An aeroengine high pressure turbine is studied to build its simplified model in Figure 1(a). 


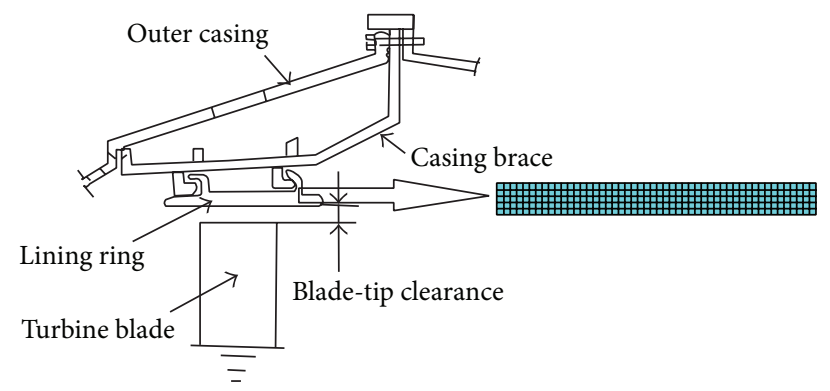

(a)

(b)

FIGURE 1: High pressure turbine structure (a) BTRRC sketch and (b) FE model of turbine casing.

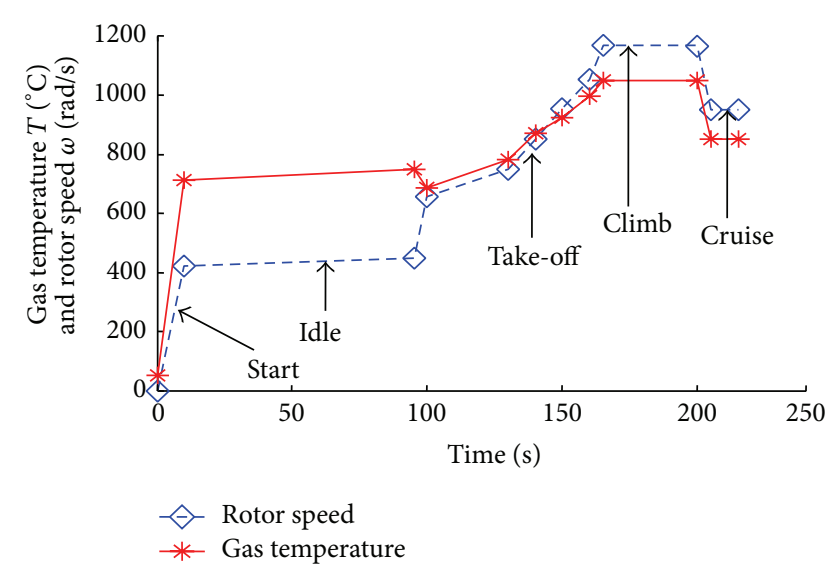

FIGURE 2: Load spectrums for aeroengine.

Detailed studies of turbine stator system have been conducted $[1,3,25-27]$. The casing structure is modeled as a ringlike structure made up of a series of arcs joined together [27]. The lining ring of casing is a sensitive component so that its expansion and contraction lead to the variation of casing radial deformation and blade-tip clearance. Hence, the inner lining ring is only studied and simplified as a cylindrical structure. For the convenient calculation, an axial cross-section of the axisymmetric FE model is extracted as an interesting submodel as shown in Figure 1(b). The left axial degree of freedom of the submodel is restrained to prevent the axial motion. The inner surface of FE model is exposed to turbine inlet hot gas, and the outside surface is exposed to compressor discharge air.

3.2. Parameters Selection. Under operating condition, rotor speed $\omega$ and gas temperature $T$ change with time (timevarying), and the flight profile parameter of which is selected for aeroengine load spectrums [1-3,27] as shown in Figure 2. Thermal expansion coefficients $\kappa$ and thermal conductivities $\lambda$ possess obvious nonlinearity as listed in Table 1 . Hence, the inner and outer surface temperatures of casing are timevarying and are predetermined by gas temperature $T$ and corresponding convection heat transfer coefficients [28-30]. The geometric parameters of casing are predetermined in manufacturing processes, which possesses obvious randomness. Because of only analyzing the radial deformation of casing, the thickness $h$ is regarded in casing optimization design. For analysis convenience, the maximum values of aforementioned nonlinear and dynamic parameters were selected as random variables by the extremum selection method [31]. Therefore, the distribution characteristics of these parameters were listed in Table 2.

3.3. Probabilistic Analysis Approach. For turbine casing radial deformation analysis, output response and input random variables are denoted by $Y$ and $\mathbf{x}$, respectively. If the predetermined minimum deformation is $\delta_{0}$, the limit state function with (10) is

$$
\begin{aligned}
H(\mathbf{x}) & =Y(\mathbf{x})-\delta_{0} \\
& =\operatorname{Max}_{j} f\left(\mathbf{x}_{j}\right)-\delta_{0} \\
& =\operatorname{Max}_{j}\left\{\sum_{\mathbf{x}_{i j} \in S V}\left(\bar{a}_{i j}-\bar{a}_{i j}^{*}\right) \psi\left(\mathbf{x}_{j}, \mathbf{x}_{i j}\right)+\bar{b}_{j}\right\}-\delta_{0} .
\end{aligned}
$$

From (11), $H \leq 0$ indicates the system failure while $H \geq$ 0 denotes that the system is secure. According to the limit state function, the fact that the maximum casing radial deformation $Y(\mathbf{x})$ within time domain $[0, T]$ is greater than the permissible maximum deformation $\delta_{0}$ is able to ensure the safety of aeroengine. This is also the reason why the presented T-LSSVM method is applied in the dynamic probabilistic optimization of turbine casing radial deformation.

When $\boldsymbol{\mu}=\left[\mu_{1}, \mu_{2}, \ldots, \mu_{r}\right], \mathbf{D}=\left[D_{1}, D_{2}, \ldots, D_{r}\right]$ and the random variables $x_{1}, x_{2}, \ldots, x_{r}$, are mutually independent, $H, \mathbf{x}, \boldsymbol{\mu}$, and $\mathbf{D}$ meet

$$
\begin{gathered}
E\left(x_{i}^{2}\right)=E^{2}\left(x_{i}\right)+D\left(x_{i}\right)=\mu_{i}^{2}+D_{i}, \\
E\left(x_{i} x_{j}\right)=E\left(x_{i}\right) E\left(x_{j}\right)=\mu_{i} \mu_{j}, \\
D\left(x_{i}^{2}\right)=4 \mu_{i}^{2} D_{i}+2 D_{i}, \\
D\left(x_{i} x_{j}\right)=\mu_{i}^{2} D_{j}+\mu_{j}^{2} D_{i}+D_{i} D_{j}, \\
E[H]=\mu_{H}\left(\mu_{1}, \mu_{2}, \ldots, \mu_{r}, D_{1}, D_{2}, \ldots, D_{r}\right), \\
D[H]=D_{H}\left(\mu_{1}, \mu_{2}, \ldots, \mu_{r}, D_{1}, D_{2}, \ldots, D_{r}\right),
\end{gathered}
$$

where $E(\cdot)$ and $D(\cdot)$ are the functions of mean value and variance function, respectively; $\mu_{H}$ and $D_{H}$ are the mean value and variance function functions of the limit state function $H ; i, j=1,2, \ldots, r ; r$ is the number of random variables.

When $H$ obeys a normal distribution, its reliability index $\beta$ and reliability degree $R$, respectively, are

$$
\begin{aligned}
& \beta=\frac{\mu_{H}}{\sqrt{D_{H}}}, \\
& R=\Phi(\beta) .
\end{aligned}
$$


TABLE 1: Heat conductivity and expansion coefficient under different temperature (reference $20^{\circ} \mathrm{C}$ ).

\begin{tabular}{|c|c|c|c|c|c|c|c|c|c|c|}
\hline Temperature, ${ }^{\circ} \mathrm{C}$ & 100 & 200 & 300 & 400 & 500 & 600 & 700 & 800 & 900 & 1000 \\
\hline Thermal conductivity $\lambda, \mathrm{W} \cdot \mathrm{m}^{-1} \cdot{ }^{\circ} \mathrm{C}^{-1}$ & 14.6 & 1606 & 18.0 & 19.4 & 20.8 & 22.4 & 23.8 & 25.0 & 26.6 & 27.8 \\
\hline Thermal expansion coefficient $\kappa, 10^{-5 \circ} \mathrm{C}^{-1}$ & 1.28 & 1.35 & 1.43 & 1.50 & 1.55 & 1.61 & 1.70 & 1.76 & 1.80 & 1.87 \\
\hline
\end{tabular}

TABLE 2: Numerical characteristic of extremum random variables.

\begin{tabular}{lccccc}
\hline Types & Random variables & Mean values & StDev. & Lower bound & Upper bound \\
\hline Casing thickness, $10^{-3} \mathrm{~m}$ & $h$ & 3 & 0.06 & 2.97 & 3.03 \\
Gas temperature, ${ }^{\circ} \mathrm{C}$ & $T$ & 1050 & 31.5 & 1034 & 1800 \\
Convection heat transfer coefficients, $\mathrm{W} \cdot \mathrm{m}^{-2} \cdot{ }^{\circ} \mathrm{C}^{-1}$ & $\alpha$ & 6000 & 180 & 5910 & 6090 \\
Thermal expansion coefficient, $10^{-5}{ }^{\circ} \mathrm{C}^{-1}$ & $\kappa$ & 1.87 & 0.0561 & 1.842 & 1.898 \\
Thermal conductivity, $\mathrm{W} \cdot \mathrm{m}^{-1} \cdot{ }^{\circ} \mathrm{C}^{-1}$ & $\lambda$ & 27.8 & 0.834 & 27.4 \\
\hline
\end{tabular}

3.4. Optimization Model of Turbine Casing Radial Deformation. Constraints for turbine casing optimization consist of design variable constraints and performance constraints. The design variables constraint change ranges. We consider the reliability degree of casing radial deformation as the performance constraints to minimize the maximum value of casing radial deformation. The reliability degree is a function of random parameters. Hence, under the permissible reliability degree $R_{0}$ and the limited variable ranges, the reliability optimization model is formulated as

$$
\begin{array}{ll}
\min \quad & \left(H\left(\mathbf{x}^{k}\right)\right) \\
& =E\left(Y\left(\mathbf{x}^{k}\right)-\delta_{0}^{k}\right) \\
& =E\left(\operatorname{Max}_{j} f\left(\mathbf{x}_{j}^{k}\right)-\delta_{0}^{k}\right) \\
& =E\left(\operatorname{Max}_{j}\left\{\sum_{\mathbf{x}_{i j}^{k} \in S \mathrm{~V}}\left(\bar{a}_{i j}^{k}-\bar{a}_{i j}^{k *}\right) \psi\left(\mathbf{x}_{j}^{k}, \mathbf{x}_{i j}^{k}\right)+\bar{b}_{j}^{k}\right\}\right. \\
\text { s.t. } \left.\quad-\delta_{0}^{k}\right) & \frac{\mu_{\delta_{0}^{k}}-\mu_{Y_{k}}}{\sqrt{\delta_{\delta_{0}^{k}}^{2}+\delta_{Y_{k}}^{2}} \geq \Phi^{-1}\left(R_{0}\right),} \\
g\left(\mathbf{x}_{k}, t\right)=0, \\
\quad \mathbf{x}_{k}=(h, T, a, k, \lambda) \in[\mathbf{a}, \mathbf{b}], \\
i=1,2,3, \ldots
\end{array}
$$

in which $k$ is the $k$ th circular computation; $\mathbf{x}_{k}$ is the design variable vector of the $k$ th loop; $t$ is the time; $\mu_{\delta_{0}^{k}}$ and $\delta_{\delta_{0}^{k}}$ are the allowable mean and standard deviation of $\delta_{0, k}$ after the $k$ th loop, respectively; $\mu_{Y_{k}}$ and $\delta_{Y_{k}}$ are the mean and standard deviation of output response after the $i$ th loop; $\Phi^{-1}(\cdot)$ is the inverse normal distribution function; $g\left(\mathbf{x}_{k}, t\right)$ is the equality constraint of the $i$ th loop that the design variables should meet; $\mathbf{a}$ and $\mathbf{b}$ are the lower bound and upper bound of random variables; $R_{0}$ is the predetermined reliability degree, respectively.

In (14), the correlative parameters $\left(\mu_{Y_{k}}, \delta_{Y_{k}}, g\left(\mathbf{x}_{k}, t\right), R_{0}\right)$ are denoted by random design variables $\mathbf{x}_{k}$ and the expression of output response $Y\left(\mathbf{x}_{k}\right)$ is gained based on these design variables. In line with Monte Carlo (MC) method, the mean value $\mu_{\mathbf{x}_{k}}$ and standard deviation $\delta_{\mathbf{x}_{k}}\left(\delta_{\mathbf{x}_{k}}=v_{\mathbf{x}} \cdot \mu_{\mathbf{x}_{k}}, v_{\mathbf{x}}\right.$ is the variation coefficient of design variables) of design variable vector rather than the mean value $\mu_{Y_{i}}$ and standard deviation $\delta_{Y_{k}}$ are inputted into (14) to obtain the mean value model of structural reliability optimal design.

3.5. Basic Idea of Optimization Design for Casing Radial Deformation. The FE method and T-LSSVM are to be adopted in the probabilistic optimal design of casing radial deformation. In this analysis process, the optimization design needs many times loop calculations. The flowchart of structural probabilistic optimization using T-LSSVM is shown in Figure 3.

\section{Experimental Study}

The experimental study for assessing the proposed method consists of two stages. During the first stage, two numerical test functions with nonlinearity or dynamic are employed in assessing the accuracy and efficiency of T-LSSVM through the comparison of the LSSVM [18]. The second stage is the reliability optimization and verification of casing radial deformation with the T-LSSVM. All calculations are performed on the same computer.

4.1. Numerical Experiment. The first test problem, denoted by $\mathrm{P} 1$, is the Haupt function with nonlinearity [5], defined as

$$
\begin{aligned}
\min f(x)= & 2-0.01\left(x_{2}-x_{1}^{2}\right)^{2}+\left(1-x_{1}\right)^{2} \\
& +2\left(2-x_{2}\right)^{2}+7 \sin \left(0.5 x_{1}\right) \sin \left(0.7 x_{1} x_{2}\right),
\end{aligned}
$$

where $x_{1}, x_{2} \in[0,5]$. It has three local optimal solutions. One of them is the global solution with a value of -1.4565 as $x=$ $(2.5044,2.5778)$. 
TABLE 3: Numerical experiment results using LSSVM and T-LSSVM.

\begin{tabular}{lccccc}
\hline Problem & Method & Objective value & Relative error & Sample number & Elapsed time, $s$ \\
\hline \multirow{2}{*}{ P1 } & LSSVM & -1.31972 & $9.391 \%$ & 31 & 47.1 \\
& T-LSSVM & -1.45591 & $0.041 \%$ & 29 & 53.7 \\
\hline \multirow{2}{*}{ P2 } & LSSVM & 149.68781 & $5.136 \%$ & 61 & 236.4 \\
& T-LSSVM & 158.18375 & $0.567 \%$ & 63 & 269.7 \\
\hline
\end{tabular}

TABLE 4: Optimization results of three methods (RSM, ERSM, and MC method).

\begin{tabular}{|c|c|c|c|c|c|}
\hline \multirow{2}{*}{ Method } & \multicolumn{2}{|c|}{ Fit response surface model } & \multicolumn{3}{|c|}{ Test response surface model } \\
\hline & Sample number & Fit time, s & Sample number & Computational time, s & Precision \\
\hline MC method & - & - & 200 & 2014 & 1 \\
\hline LSSVM & 49 & 815.3 & 200 & 9.972 & 0.95321 \\
\hline T-LSSVM & 49 & 638.7 & 200 & 8.561 & 0.98931 \\
\hline
\end{tabular}

The second test problem, denoted by $\mathrm{P} 2$, is a nonlinear dynamic function with semi-infinite and multivariable constraints, defined as

$$
\begin{array}{ll}
\max & f(x)=10 x_{1}+4.4 x_{2}^{2}+2 x_{3} \\
\text { s.t. } & 0.5 x_{3}^{2}-x_{2}^{2} \geq 3 \\
& x_{1}+4 x_{2}+5 x_{3} \leq 32 \\
& x_{1}+3 x_{2}+2 x_{3} \leq 29 \\
& x_{1} \geq 0, \quad x_{2} \geq 0, \quad x_{3} \geq 0,
\end{array}
$$

where the semi-infinite time-varying constraints $K_{i}(x, t)(i=$ $1,2)$ are

$$
\begin{aligned}
& K_{1}(x, t)= \sin \left(x_{1} t\right) \cos \left(x_{2} t\right) \\
&-\frac{1}{100}(t-50)^{2}-\sin \left(x_{3} t\right)-x_{3}-1 \leq 0 \\
& K_{2}(x, t)= \sin \left(x_{2} t\right) \cos \left(x_{1} t\right) \\
&-\frac{1}{100}(t-50)^{2}-\sin \left(x_{3} t\right)-x_{3}-1 \leq 0 \\
& t \in[0,100 \mathrm{~s}] .
\end{aligned}
$$

It has an optimal solution $x=(4.2302,5.0559,1.5093)$ with a function value of 157.7923 .

LSSVM and T-LSSVM are used to conduct the two problems. The comparisons of results are listed in Table 3.

As shown in Table 3, the objective values of LSSVM are -1.31972 and 149.68781 for two problems and their corresponding relative errors are $9.391 \%$ and $5.136 \%$, respectively. However, the objective values of T-LSSVM are -1.45591 and 158.18375 , respectively, which are quite close to the theoretical optimal values (the relative errors are only $0.041 \%$ and $0.567 \%$ ). The number of needed samples and elapsed time for two test problems with T-LSSVM are consistent with LSSVM. Obviously, T-LSSVM has higher computational accuracy with keeping computational efficiency in nonlinear dynamic optimization. Hence, T-LSSVM provides a useful insight into the optimum control-based nonlinear dynamic probabilistic optimization of turbine casing radial deformation.

\subsection{Optimization Design of Turbine Casing Radial Deformation}

4.2.1. Dynamic Deterministic Analysis. Base on the FE model of casing and MC method, the dynamic deterministic analysis of casing radial deformation was fulfilled by the thermosetting coupling analysis. Casing time-varying radial deformation is shown in Figure 4. Obviously, turbine casing deformation reaches the maximum at $t=185 \mathrm{~s}$ approximately, and the maximum radial deformation is $8.651 \times 10^{-4} \mathrm{~m}$.

In this dynamic analysis, the maximum value of casing radial deformation determines whether the system is safe during the whole time domain $[0,215 \mathrm{~s}]$, because the limit state function is secure in the whole analysis process if the extremum of casing radial deformation in $[0,215 \mathrm{~s}]$ is smaller than the predetermined deformation $\delta_{0}$. Therefore, the ERSM is suitable for the probabilistic optimal design of casing radial deformation.

4.2.2. Fit and Validate ERSFs. A small quantity of samples of each random variable was extracted and afterwards inputted into the FE model of turbine casing to gain the corresponding extremum output responses. The obtained many groups of samples are called as fitting samples with input samples and output samples and are used to construct LSSVM and TLSSVM models. The required sample number and fitting time of LSSVM and T-LSSVM models are drawn in Table 4. Afterwards 200 groups of testing samples were extracted and were used to test LSSVM and T-LSSVM models. The analysis results are shown in Table 4 .

As shown in Table 4, in the stage of fitting response surface, T-LSSVM costs $638.2 \mathrm{~s}$, which consumes less runtime than LSSVM (815.3 s) although the same number of samples is needed to fit response surface models. From the test results of two response surface models by 200 times simulations, it is revealed that T-LSSVM saves computing time $1.411 \mathrm{~s}$ and improves computational precision $3.611 \%$ relative to LSSVM, 
TABLE 5: Optimization results of casing radial deformation based on T-LSSVM.

\begin{tabular}{lcccccc}
\hline $\begin{array}{c}\text { Before } \\
\text { optimization }\end{array}$ & $\begin{array}{c}\text { Offer } \\
\text { optimization }\end{array}$ & $\begin{array}{c}\text { Reduced } \\
\text { deformation }\end{array}$ & Reliability degree & Function evaluations & Iterations & Time elapsed, s \\
\hline 8.651 & 7.865 & 0.786 & 0.9956 & 1591 & 37 & 8237 \\
\hline
\end{tabular}

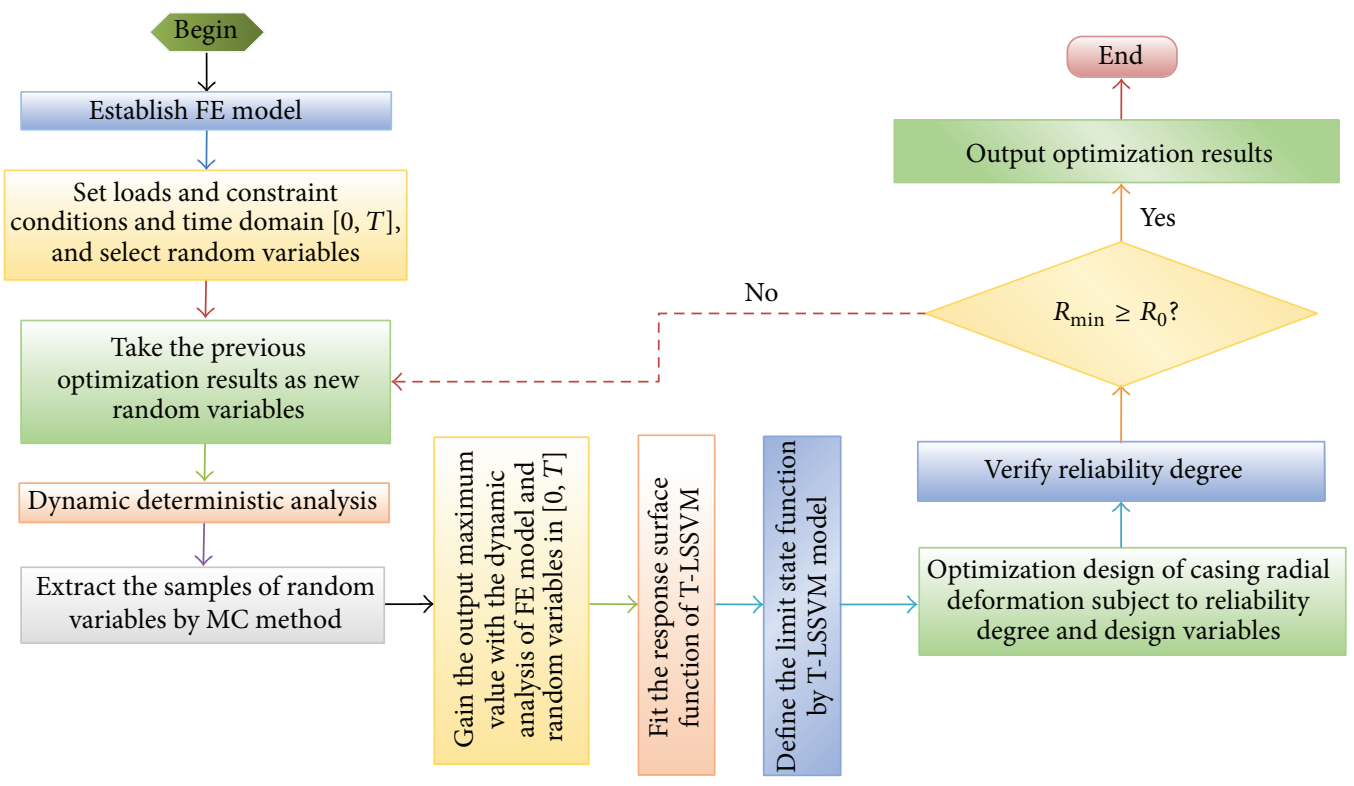

FIgURE 3: Procedure of dynamic reliability optimal design analysis with T-LSSVM.

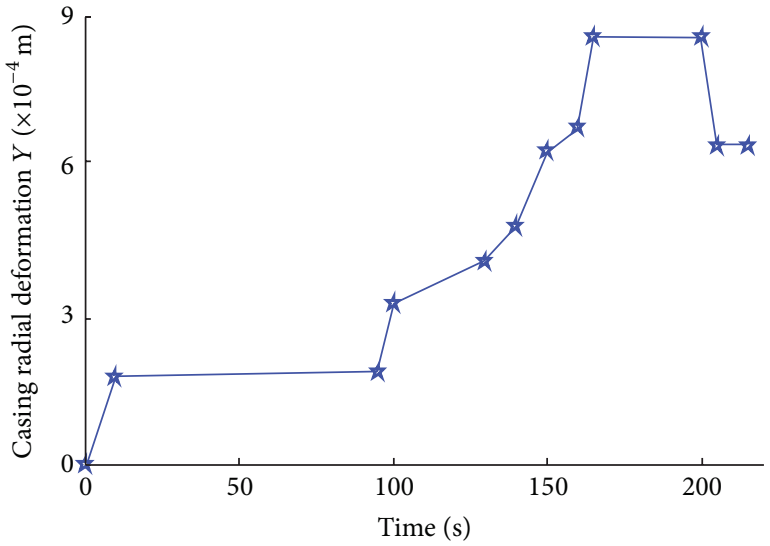

FIGURE 4: Variation of casing radial deformation $Y$.

which demonstrates the high computational efficiency and accuracy of T-LSSVM.

\subsubsection{Probabilistic Optimal Design for Turbine Casing Radial} Deformation. In the optimization model (14) of casing radial deformation, it is presumed that the reliability degree $R_{0}$ is 0.99 and the minimum allowable value $\delta_{0}$ is $8 \times 10^{-4} \mathrm{~m}$. The reliability optimal design of casing radial deformation is enforced with the proposed T-LSSVM through 10000 times simulations in each iteration. The probabilistic distributions

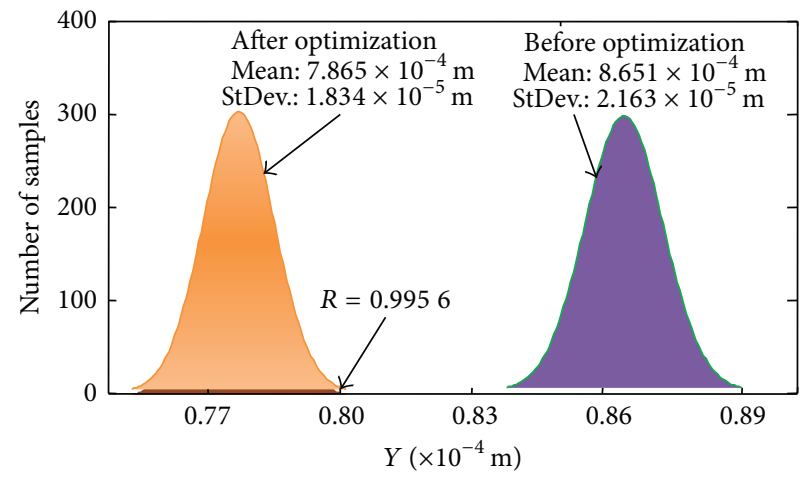

FIGURE 5: Probability distributions of maximum output response $Y$ $\left(\times 10^{-4} \mathrm{~m}\right)$ before and after optimization.

of maximum output response $Y$ before and after optimization were shown in Figure 5. From the optimization, the optimal solution of T-LSSVM is $\mathbf{x}^{*}=\left(3 \times 10^{-3}, 1034.3,5910,1.851\right.$, and 27.73). The objective values, computational accuracy, optimal time, iterations, and function counter are drawn in Table 5. The distribution of casing radial deformation before and after optimization is shown in Figure 6.

As shown in the Figure 5, the variation of casing radial deformation (or extremum output response) obeys a normal distribution. The mean and standard deviation obtained by T-LSSVM are $7.865 \times 10^{-4} \mathrm{~m}$ and $1.834 \times 10^{-5} \mathrm{~m}$. 


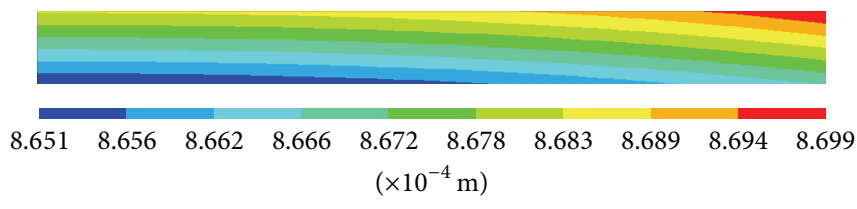

(a)

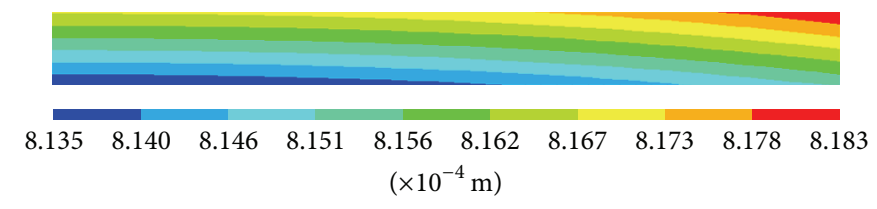

(b)

FIGURE 6: Casing radial deformation distribution (a) before and (b) after optimization.

As shown in Table 5 and Figures 5 and 6 , the casing radial deformation after optimization with T-LSSVM reduces by $7.86 \times 10^{-5} \mathrm{~m}$ with the reliability degree $R=0.9956$ to that before optimization, when the maximum permissible radial deformation $\delta_{0}=8 \times 10^{-4} \mathrm{~m}$, which pledges the reliability and work efficiency of blade-tip clearance. The solutions are promising to preferably control blade-tip clearance and develop the high performance and high reliability of gas turbine. The computational efficiency and precision of TLSSVM are also acceptable for structural nonlinear dynamic probabilistic optimization.

\section{Conclusions}

To seek the optimum control of casing radial deformation with thermal stress, pressure differential, and nonlinear material properties, a probabilistic optimization method (TLSSVM) is proposed. Some conclusions are summarized as follows.

(1) The mathematical model of T-LSSVM method is established for structural dynamic probabilistic optimization.

(2) The optimization model of turbine casing radial deformation is constructed with T-LSSVM.

(3) The proposed T-LSSVM is promising to improve computational precision with the acceptable calculation efficiency.

(4) The establishment of T-LSSVM is promising to significantly reduce the total computational cost due to a small number of expensive FE analyses.

(5) Desirable radial deformation and reasonable reliability are obtained for turbine casing design; meanwhile, T-LSSVM is proved to be feasible and effective in nonlinear dynamic optimization.

Despite current work demonstrates the potential of TLSSVM, more studies should be performed in our future work to validate T-LSSVM in other complex design optimization problems.

\section{Conflict of Interests}

The authors declare that there is no conflict of interests regarding the publication of this paper.

\section{Acknowledgment}

The authors would like to thank the National Natural Science Foundation of China which cosupported the paper (Grant nos. 51175017 and 51275024).

\section{References}

[1] S. B. Lattime and B. M. Steinetz, "High pressure turbine clearance control system: current practices and future directions," Journal of Propulsion and Power, vol. 20, no. 2, pp. 302-311, 2004.

[2] A. E. Nielsen, C. W. Moll, and S. Staudacher, "Modeling and validation of the thermal effects on gas turbine transients," Journal of Engineering for Gas Turbines and Power, vol. 127, no. 3, pp. 564-572, 2005.

[3] G. C. Bai and C. W. Fei, "Distributed collaborative response surface method for mechanical dynamic assembly reliability design," Chinese Journal of Mechanical Engineering, vol. 26, no. 6, pp. 1160-1168, 2013.

[4] D. Hu, R. Wang, and Z. Tao, "Probabilistic design for turbine disk at high temperature," Aircraft Engineering and Aerospace Technology, vol. 83, no. 4, pp. 199-207, 2011.

[5] Z. J. Huang, C. G. Wang, J. Chen, and H. Tian, "Optimal design of aeroengine turbine disc based on kriging surrogate models," Computers and Structures, vol. 89, no. 1-2, pp. 27-37, 2011.

[6] L. Witek, M. Orkisz, P. Wygonik, D. N. Musili, and T. Kowalski, "Fracture analysis of a turbine casing," Engineering Failure Analysis, vol. 18, no. 3, pp. 914-923, 2011.

[7] S. A. Meguid, P. S. Kanth, and A. Czekanski, "Finite element analysis of fir-tree region in turbine discs," Finite Elements in Analysis and Design, vol. 35, no. 4, pp. 305-317, 2000.

[8] W. Song, A. Keane, J. Rees, A. Bhaskar, and S. Bagnall, "Turbine blade fir-tree root design optimisation using intelligent CAD and finite element analysis," Computers and Structures, vol. 80, no. 24, pp. 1853-1867, 2002.

[9] B. Farshi, H. Jahed, and A. Mehrabian, "Optimum design of inhomogeneous non-uniform rotating discs," Computers \& Structures, vol. 82, no. 9-10, pp. 773-779, 2004.

[10] L. Witek, "Failure analysis of turbine disc of an aero engine," Engineering Failure Analysis, vol. 13, no. 1, pp. 9-17, 2006.

[11] W. Shyy, N. Papila, R. Vaidyanathan, and K. Tucker, "Global design optimization for aerodynamics and rocket propulsion components," Progress in Aerospace Sciences, vol. 37, no. 1, pp. 59-118, 2001.

[12] A. I. J. Forrester and A. J. Keane, "Recent advances in surrogatebased optimization," Progress in Aerospace Sciences, vol. 45, no. 1-3, pp. 50-79, 2009.

[13] G. G. Wang, "Adaptive response surface method using inherited Latin hypercube design points," Journal of Mechanical Design, Transactions of the ASME, vol. 125, no. 2, pp. 210-220, 2003. 
[14] O. Yeniay, R. Unal, and R. A. Lepsch, "Using dual response surfaces to reduce variability in launch vehicle design: a case study," Reliability Engineering and System Safety, vol. 91, no. 4, pp. 407-412, 2006.

[15] M. E. Kartal, H. B. Başaĝa, and A. Bayraktar, "Probabilistic nonlinear analysis of CFR dams by MCS using response surface method," Applied Mathematical Modelling, vol. 35, no. 6, pp. 2752-2770, 2011.

[16] Q. Lü and B. K. Low, "Probabilistic analysis of underground rock excavations using response surface method and SORM," Computers and Geotechnics, vol. 38, no. 8, pp. 1008-1021, 2011.

[17] C.-W. Fei and G.-C. Bai, "Distributed collaborative probabilistic design for turbine blade-tip radial running clearance using support vector machine of regression," Mechanical Systems and Signal Processing, vol. 49, pp. 196-208, 2014.

[18] Z. W. Guo and G. C. Bai, "Application of least squares support vector machine for regression to reliability analysis," Chinese Journal of Aeronautics, vol. 22, no. 2, pp. 160-166, 2009.

[19] A. Basudhar and S. Missoum, "Adaptive explicit decision functions for probabilistic design and optimization using support vector machines," Computers and Structures, vol. 86, no. 19-20, pp. 1904-1917, 2008.

[20] J. Tian and H. Gu, "Anomaly detection combining one-class SVMs and particle swarm optimization algorithms," Nonlinear Dynamics, vol. 61, no. 1-2, pp. 303-310, 2010.

[21] C. Fei and G. Bai, "Nonlinear dynamic probabilistic analysis for turbine casing radial deformation using extremum response surface method based on support vector machine," Journal of Computational and Nonlinear Dynamics, vol. 8, no. 4, Article ID 041004, 2013.

[22] C. Bucher and T. Most, "A comparison of approximate response functions in structural reliability analysis," Probabilistic Engineering Mechanics, vol. 23, no. 2-3, pp. 154-163, 2008.

[23] A. A. Giunta and L. T. Watson, "A comparison of approximation modeling techniques: polynomial versus interpolating models," in Proceedings of the 7th AIAA/USAF/NASA/ISSMO Symposium on Multidisciplinary Analysis and Design, St. Louis, Mo, USA, September 1998.

[24] C. W. Fei and G. C. Bai, "Wavelet correlation feature scale entropy and fuzzy support vector machine approach for aeroengine whole-body vibration fault diagnosis," Shock and Vibration, vol. 20, no. 2, pp. 341-349, 2013.

[25] C. W. Fei, G. C. Bai, and T. Chao, "Extremum response surface method for casing radial, deformation probabilistic analysis," Journal of Aerospace Computing, Information and Communication, vol. 10, no. 1, pp. 47-52, 2013.

[26] C. W. Fei and G. C. Bai, "Distributed collaborative extremum response surface method for mechanical dynamic assembly reliability analysis," Journal of Central South University, vol. 20, no. 9, pp. 2414-2422, 2013.

[27] J. A. Kypuros and K. J. Melcher, "A reduced model for prediction of thermal and rotational effects on turbine tip clearance," Tech. Rep. NASA/ TM-2003-212226, 2003.

[28] Y. W. Kim, J. P. Downs, F. O. Soechting, W. Abdel-Messeh, G. D. Steuber, and S. Tanrikut, "A summary of the cooled turbine blade tip heat transfer and film effectiveness investigations performed by Dr. D.E. Metzger," ASME Journal of Turbomachinery, vol. 117, no. 1, pp. 1-11, 1995.

[29] Y. W. Kim and D. E. Metzger, "Heat transfer and effectiveness on film cooled turbine blade tip models," Journal of Turbomachinery, vol. 117, no. 1, pp. 12-21, 1995.
[30] R. Goldstein, "Film cooling," Advances in Heat Transfer, vol. 7, pp. 321-379, 1971.

[31] C. W. Fei and G. C. Bai, "Extremum selection method of random variable for nonlinear dynamic reliability analysis of turbine blade deformation," Propulsion and Power Research, vol. 1, no. 1, pp. 58-63, 2012. 

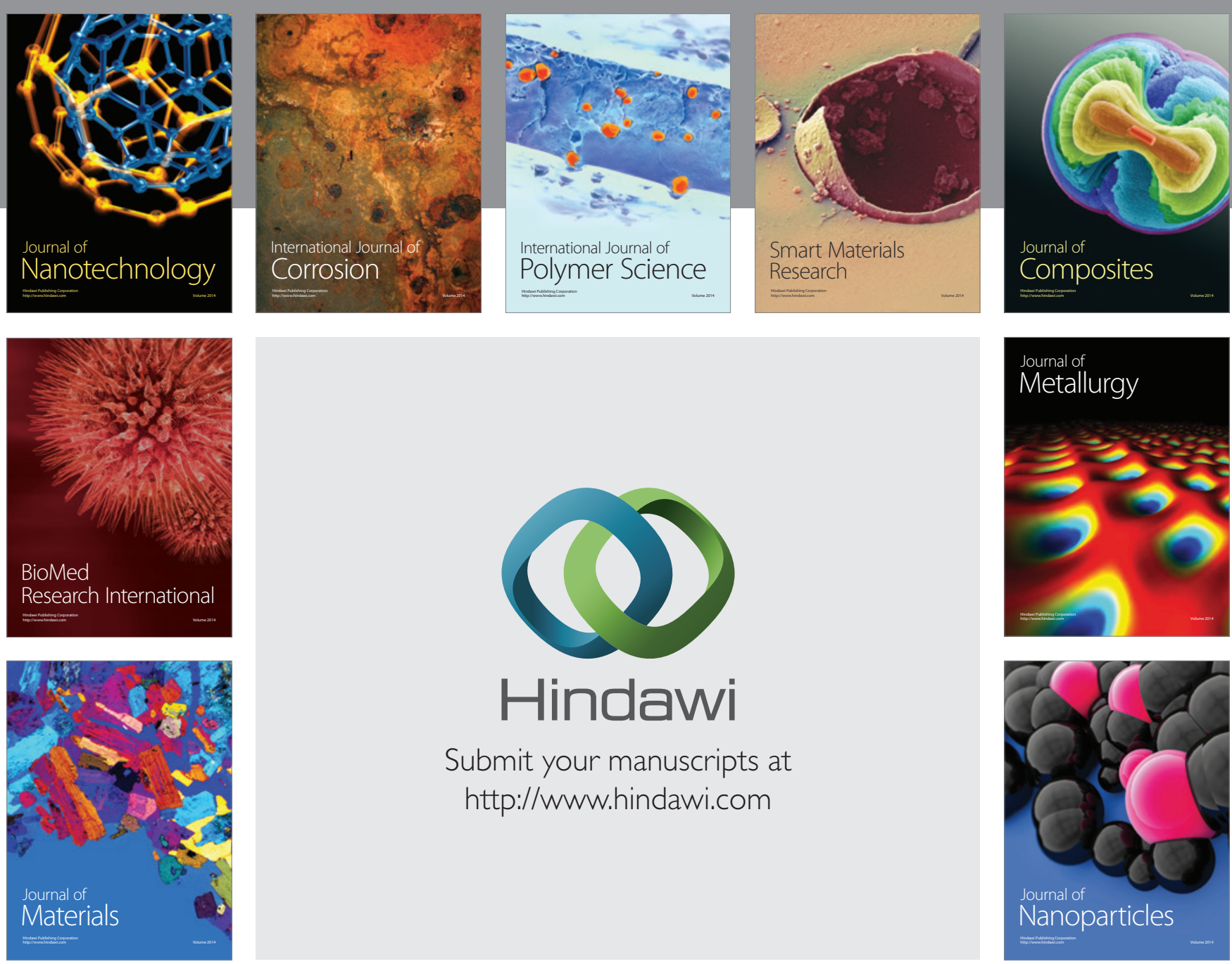

Submit your manuscripts at http://www.hindawi.com
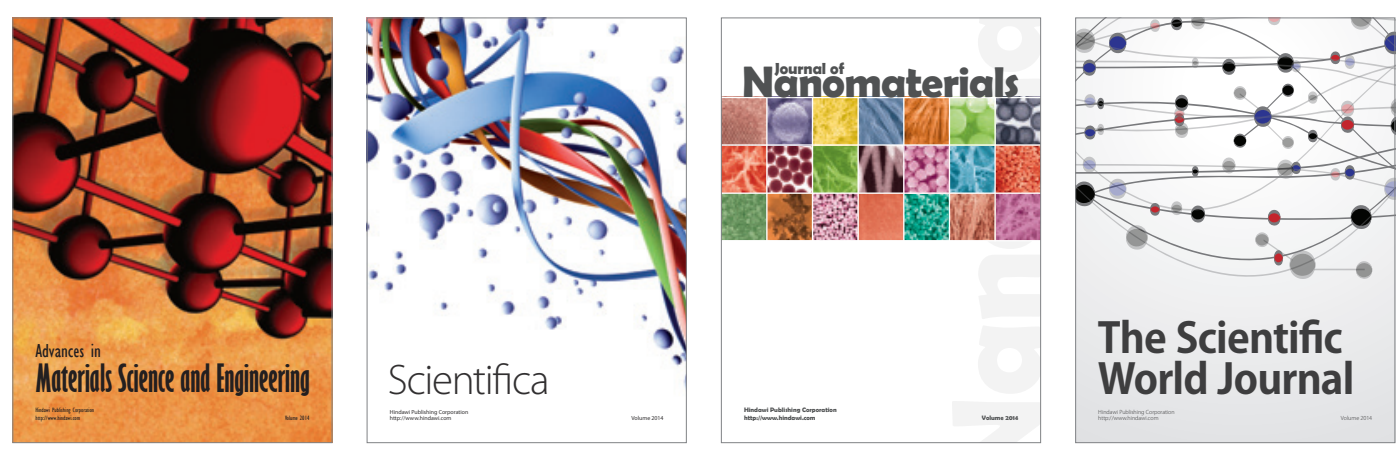

\section{The Scientific World Journal}
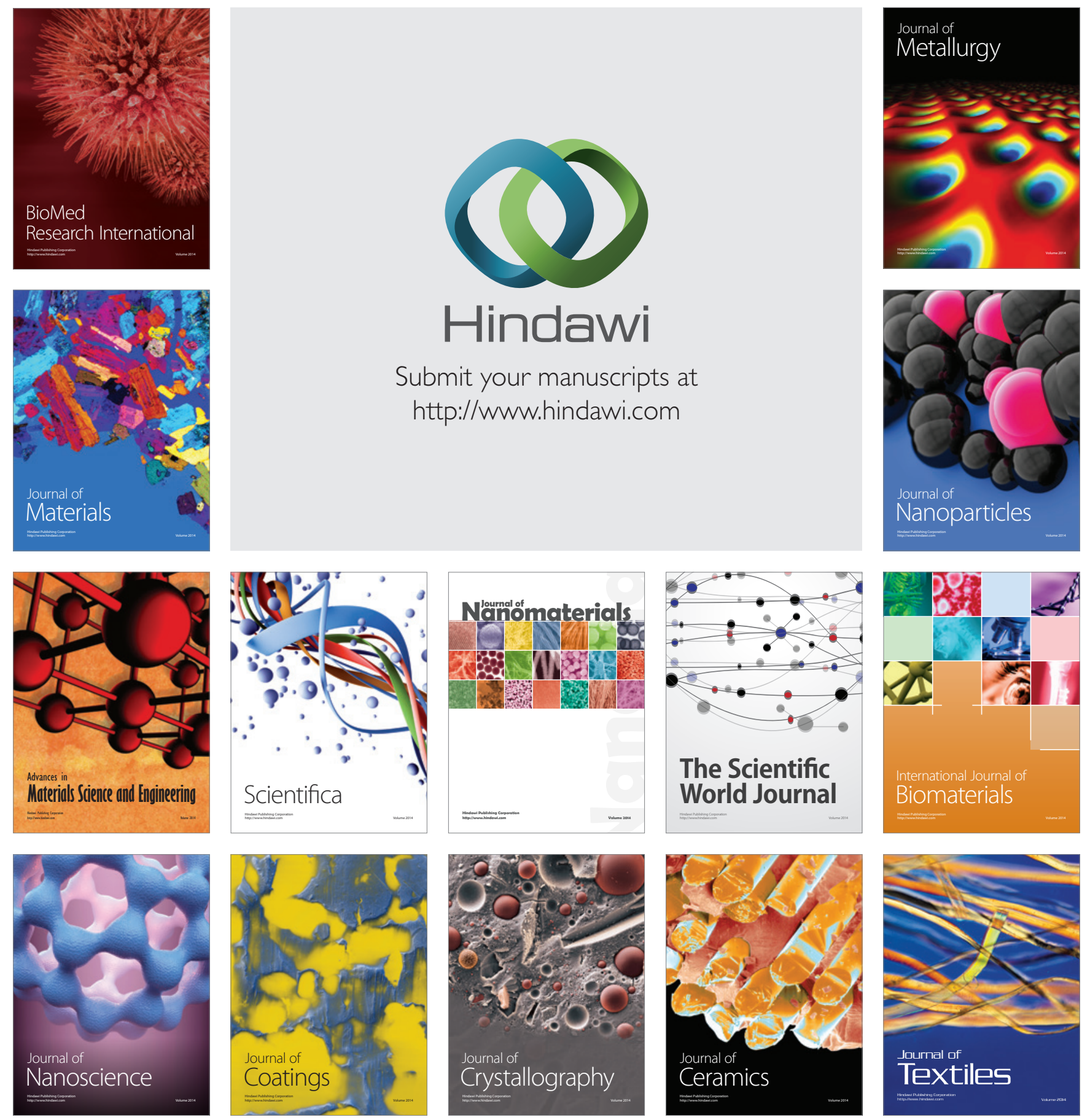